\title{
Ragna Boden
}

\section{Jakarta, 1965: Zur Rolle kommunistischer Parteien in der Dritten Welt}

Jakarta, 8. Oktober 1965. In der indonesischen Hauptstadt wurde das Zentralbüro der Kommunistischen Partei Indonesiens (Partai Komunis Indonesia- PKI) niedergebrannt: Die Verfolgung der Kommunisten hatte begonnen. Sie wurde vom Militär initiiert, koordiniert und ihre Ausbreitung über den gesamten Archipel gezielt vorangetrieben. ${ }^{1}$ Hunderttausende fielen den Ausschreitungen zum Opfer, darunter Parteimitglieder wie -anhänger, vermeintliche Sympathisanten und deren Familien oder einfach Denunzierte, die mit Kommunismus nichts zu tun hatten. Genaue Zahlen der Ermordeten, Verletzten und anderweitig Geschädigten sind nicht mehr zu ermitteln. Seriöse Schätzungen reichen von einer halben bis zu einer Million. ${ }^{2}$ Als die massivste Welle der Gewalt im März 1966 abgeebbt war, war die ehemals drittgrößte kommunistische Partei der Welt zerschlagen und verboten, ihre Führung umgebracht oder verhaftet. Die Ereignisse, die im Zusammenhang mit den Kommunistenverfolgungen stehen, hatten Auswirkungen bis in die Staatsspitze: Der erste Präsident des unabhängigen Indonesiens, Sukarno, wurde 1966/67, nach mehr als 20 Jahren im Amt, durch einen Vertreter der Armee, General Suharto, verdrängt. Damit hatte das Militär den seit Jahren schwelenden indonesischen Machtkampf zwischen Streitkräften, Präsident und Kommunistischer Partei für sich entschieden. ${ }^{3}$ Suhartos Militärregime wurde in den folgenden Jahrzehnten vom Westen umworben und bestimmte die Geschicke des Staates bis 1998. Die Volksrepublik China und die Sowjetunion hatten einen der wichtigsten (potentiellen) Bündnispartner in der sogenannten Dritten Welt verloren. Beide hatten sich um Indonesien sowohl auf staatlicher als auch auf Parteiebene bemüht und darin in zunehmend schärferer Konkurrenz zueinander gestanden. ${ }^{4}$

Auslöser der Kommunistenverfolgungen waren Ereignisse im Herbst 1965, die wiederum mit der Machtkonstellation im Archipel zusammenhingen. ${ }^{5}$ Die zeitweise schwache Gesundheit des über 60-jährigen Sukarno, der Anfang August 1965 bei einem öffentlichen Auftritt zusammengebrochen war, löste Spekulationen über seine Nachfolge aus. Sukarno hatte die Republik Indonesien (RI) seit der Ausrufung der Unabhängigkeit von den Nie-

\footnotetext{
${ }^{1} \mathrm{Zu}$ den Mechanismen vgl. Robert Cribb/Colin Brown, Modern Indonesia. A History since 1945, London/New York 1995, S. 104f.

${ }^{2}$ Für eine Übersicht vgl. Cribb/Brown, Modern Indonesia, S. 106; Robert Cribb, Introduction: Problems in the Historiography of the Killings in Indonesia, in: Ders. (Hrsg.), The Indonesian Killings of 1965-1966: Studies from Java and Bali, Clayton 1990, S. 1-43, bes. S. 12; Benedict R. O'G. Anderson, Scholarship on Indonesia and Raison d'État: Personal Experience, in: Indonesia 62 (October 1996), S. 1-18, bes. S. 1; William R. Liddle, Leadership and Culture in Indonesian Politics, Sydney 1996, S. 95; Bernhard Dahm, Indonesien, in: Bernhard Dahm/Roderich Ptak (Hrsg.), Südostasien-Handbuch, München 1999, S. 229-250, hier S. 241; A. Y. Drugov, Indonesia, in: Southeast Asia. History - Economy - Policy, Moskau 1972, S. 212-243, hier S. 236; Guy J. Pauker, Indonesien, in: C. D. Kernig (Hrsg.), Die kommunistischen Parteien der Welt, Freiburg/Basel/Wien 1969, S. 235-242, hier S. 240.

${ }^{3}$ Zur Machtkonkurrenz vgl. Herbert Feith, President Soekarno, the Army and the Communists: The Triangle Changes Shape, in: Asian Survey 4 (August 1964), S.969-980.

${ }^{4}$ Vgl. dazu unten, S. 133.

${ }^{5}$ Für die Hintergründe vgl. Cribb/Brown, Modern Indonesia; Bernhard Dahm, History of Indonesia in the Twentieth Century, London 1971; Adrian Vickers, A History of Modern Indonesia, Cambridge 2005, S. 171.
} 
derlanden am 17. August 1945 regiert und seinem Amt - und damit sich selbst - durch Machtakkumulation eine herausragende Stellung verschafft. ${ }^{6}$ Laut Gerüchten bereitete sich die Armeeführung nun darauf vor, am 5. Oktober, dem Tag der Streitkräfte, in einem Staatsstreich die Macht an sich zu reißen. Dazu kam es nicht. In der Nacht vom 30. September auf den 1.Oktober 1965 fielen sechs ranghohe Armeegeneräle Mordanschlägen zum Opfer; ein siebtes potentielles Opfer, General Nasution, konnte entkommen. ${ }^{7}$ Eine „Bewegung 30. September" übernahm am 1. Oktober die Verantwortung für die Attentate und erklärte diese „Maßnahme“ damit, dass sie einem Militärputsch mit Unterstützung der CIA habe zuvorkommen wollen. ${ }^{8}$ Besonders brisant war die Situation dadurch, dass sich als Leiter dieser „Bewegung“ der Chef der Präsidenten-Leibgarde, Oberstleutnant Untung, ausgab. Diese Aussage nährte Spekulationen, inwieweit Sukarno selbst in die Attentate verwickelt war. Noch am 1.Oktober bildete sich unter Untungs Leitung ein „Revolutionsrat“, der Anspruch auf die interimistische Führung des Landes zum Schutze von Präsident und Republik erhob. Auf einer über den Rundfunk veröffentlichten Liste der 45 Mitglieder dieses Rates fanden sich vier Kommunisten. Die Putschisten hielten sich allerdings nur einen Tag. Schon am 2. Oktober war der Aufstand unter der Leitung des Generalmajors und späteren Präsidenten Suharto niedergeschlagen.

Hatte die PKI-Führung die Verlautbarungen der „Bewegung“ zunächst begrüßt ${ }^{9}$, so distanzierte sie sich nach dem Scheitern der Rebellion davon und erklärte, mit dem Putsch habe sie nichts zu tun. Ihrer Darstellung nach waren die Namen der Kommunisten ohne deren Einverständnis auf die Liste des „Revolutionsrates“ gesetzt worden. Diese Erklärungen verhinderten jedoch nicht, dass das Militär der PKI die Verantwortung zuwies, und dies führte letztlich zu den oben beschriebenen Verfolgungen. ${ }^{10}$

\footnotetext{
${ }^{6}$ Für die Proklamation der Unabhängigkeit vgl. Osman Raliby (Hrsg.), Documenta Historica. Sedjarah Dokumenter dari pertumbuhan dan perdjuangan negara Republik Indonesia [Geschichte der Entwicklung und des Kampfes der Republik Indonesien anhand von Dokumenten], Djakarta 1953, S. 13f. Zur Phase der sogenannten gelenkten Demokratie vgl. Herbert Feith, Dynamics of Guided Democracy, in: Ruth T. McVey (Hrsg.), Indonesia, New Haven, Conn. ${ }^{21967, ~ S . ~ 309-409 ; ~ D a n i e l ~ S . ~ L e v, ~}$ Transition to Guided Democracy: Indonesian Politics, 1957-1959, Ithaca, N. Y. 1966; J. K. Tumakakam, The Indonesian Concept of Guided Democracy, Djakarta 1959; Frederick P. Bunnell, Guided Democracy Foreign Policy: 1960-1965. President Sukarno Moves from Non-alignment to Confrontation, in: Indonesia 2 (October 1966), S. 37-76.

${ }^{7} \mathrm{Zu}$ den Ereignissen vgl. die unterschiedlichen Sichtweisen von: Sirithorn Chantrasmi, Communism in Southeast Asia, Ann Arbor, Mich. 1987, S.310; Benedict R. O'G. Anderson/Ruth T. McVey, A Preliminary Analysis of the October 1, 1965 Coup in Indonesia, Ithaca, N. Y. 1971, die der Version von der innermilitärischen Auseinandersetuung zuneigen; Arnold Brackman, The Communist Collapse in Indonesia, New York 1969, der die PKI als Verantwortliche sieht; Daniel S. Lev, Indonesia 1965: The Year of the Coup, in: Asian Survey 6 (February 1966), S. 103-110.

${ }^{8}$ Dieses und die folgenden Dokumente finden sich in: Selected Documents Relating to the „September 30th Movement" and Its Epilogue, in: Indonesia 1 (April 1966), S. 131-204. Zur Rolle der CIA bei den Kommunistenverfolgungen und den Ereignissen des 30. September vgl. David Easter, ${ }_{\text {KKeep the }}$ Indonesian Pot Boiling“: Western Covert Intervention in Indonesia, October 1965 - March 1965, in: Cold War History 5 (2005), S. 55-73; Bradley R. Simpson, Economists with Guns. Authoritarian Development and U.S.-Indonesian Relations, 1960-1968, Stanford 2008, S. 156, 189 und 193; Audrey R. Kahin/George McT[urnan] Kahin, Subversion as Foreign Policy. The Secret Eisenhower and Dulles Debacle in Indonesia, Seattle/London 1995; tendenziell exkulpierend: Paul F. Gardner, Shared Hopes, Separate Fears: Fifty Years of U.S.-Indonesian Relations, Boulder, Col. 1997.

${ }^{9}$ Editorial des Parteiorgans Harian Rakjat vom 2. 10.1965, in: Selected Documents, S. 131-204, hier S. 184 .

10 Für die unterschiedlichen Deutungen der Ereignisse vgl. die in Anm. 7 genannten Titel; außerdem Vickers, Modern Indonesia, S. 171; die offizielle Darstellung der Ereignisse durch das indonesische
} 
Die sowjetische Staats- und Parteiführung zeigte sich angesichts dieser Ereignisse unvorbereitet und reagierte sowohl spät als auch verhalten. Es gab keine entschlossene Intervention zur Rettung der PKI, der Kontakt zur PKI-Spitze wurde abgebrochen, und selbst die Aufnahme indonesischer Genossen in die sowjetischen diplomatischen Vertretungen wurde aus Rücksicht auf mögliche Schwierigkeiten unterlassen. ${ }^{11}$ Dagegen erhielt man die diplomatischen Beziehungen aufrecht und suchte den Dialog mit Suharto, um die Rückzahlung von Krediten nicht zu gefährden. Hier zeigte sich die sozialistische Supermacht von einer Seite, die weder zum eigenen Anspruch passte, das sozialistische Weltsystem zu führen, noch zu den westlichen Bedrohungsszenarien, die Moskaus Einfluss bei jedem linksorientierten Aufstand am Werke sahen. Die öffentlichen Reaktionen aus der UdSSR beschränkten sich auf Äußerungen der Empörung und Betroffenheit. ${ }^{12}$ In einer internen Unterredung mit Suharto sprach der sowjetische Botschafter von einer „starken psychologischen Reaktion“ und einem „Gefühl großer Bitterkeit“, die die Massenmorde bei der sowjetischen Bevölkerung ausgelöst hätten. ${ }^{13}$ Dass die Opfer dieser Massaker in erster Linie Kommunisten waren, führte er nicht offen aus. Deutlichere Worte gebrauchte der Vorsitzende des Ministerrats Aleksej Kosygin gegenüber dem indonesischen Außenminister, indem er die Kommunistenverfolgungen bestialisch nannte. ${ }^{14}$ Die verbalen Proteste hatten jedoch denkbar geringen Einfluss auf die neue indonesische Staatsführung und halfen den verfolgten Kommunisten nicht. Die sowjetischen Vertretungen vor Ort spielten in dieser Zeit keine rühmliche Rolle: Aus Furcht vor Übergriffen auf die eigenen Mitarbeiter brachen sie die Verbindungen zu den indonesischen Genossen ab. ${ }^{15}$ Einige wenige indonesische Kommunisten konnten fliehen und gingen ins Exil nach China, Albanien oder Moskau. ${ }^{16}$ Dort arbeiteten sie in Exilkomitees weiter, ohne jedoch noch einmal im Archipel selbst Fuß fassen zu können. Damit schloss sich für die PKI der Kreis: aus dem sowjetischen Exil waren die Aktivisten in das von den Niederländern beherrschte Land gekommen, um dort den Kommunismus einzuführen; von dort waren sie wieder in sozialistische Länder vertrieben worden. Diesmal bedeutete ihre Flucht jedoch, dass es auf lange Zeit kein sozialistisches Indonesien geben würde.

Außenministerium wandelt sich. Bis Januar 2005 war auf dessen Homepage zu lesen, dass die Kommunisten für die Morde an den Generälen verantwortlich waren. Im Jahre 2006 war dieser Passus getilgt. Vgl. Ragna Boden, The „Gestapu“ Events of 1965 in Indonesia, in: Bijdragen tot de Taal-, Land- en Volkenkunde 163 (2007), S. 507-528, hier S.507 Anm. 3. Mit wachsendem Abstand von Suhartos Amtszeit rückt das Thema allerdings zunehmend ins öffentliche Interesse.

"Vgl. den Bericht des DDR-Konsuls Kehr an das ZK der SED, 12.1.1966 (Stiftung Archiv Parteien und Massenorganisationen der DDR [im Folgenden: SAPMO-BArch], DY/30/IV A 2/20, 668, o. P., 1. Bl.); Boden, „Gestapu“.

12 Siehe hierzu die Dokumente in: V zaščitu borcov protiv reakcii i imperializma. K sobytijam v Indonezii [Zur Verteidigung der Kämpfer gegen Reaktion und Imperialismus. Zu den Ereignissen in Indonesien], 2 Bde., Moskau 1967-1969.

13 Gespräch Sytenkos mit Suharto, 29.12.1967 (Archiv Vnešnej Politiki Rossijskoj Federacii [Archiv des Außenministeriums der Russländischen Föderation, im Folgenden: AVP RF], f.91, 23-41-6, 11. 222-235, hier 1. 228).

14 „Information über den Besuch des indonesischen Außenministers in der Sowjetunion“, Bericht des DDR-Gesandten Rossmeisl aus der Botschaft in Moskau, 25.10.1966 (SAPMO-BArch, DY/30/IV A 2/20, 671, o. P., 2. Bl.).

${ }^{15}$ Bericht des DDR-Konsuls Kehr an das ZK der SED, 12.1.1966 (SAPMO-BArch, DY/30/IV A 2/20, 668 , o. P., 1. Bl.).

${ }^{16}$ „Informationsmaterial über die Kommunistische Partei Indonesiens“ (SAPMO-BArch, DY/30/IV A 2/20, 1051, o. P., 3.-4. Bl.). 


\section{Die Anfänge der kommunistischen Parteien: Die Komintern}

Die verhaltenen öffentlichen Reaktionen aus der UdSSR auf die Kommunistenverfolgungen in der RI sind nur vor dem Hintergrund der globalen Kräftekonstellationen zu verstehen. Gleichzeitig weist die Entwicklung der Moskauer Beziehungen zu den indonesischen Kommunisten viele typische Merkmale des Umgangs der sowjetischen Führung mit den kommunistischen Parteien der Entwicklungsländer generell auf. Die Geschichte der PKI ist in vielerlei Hinsicht bezeichnend für die KPs postkolonialer Staaten und Regionen. ${ }^{17}$ Sie war 1920 noch vor der KP Chinas ${ }^{18}$ gegründet worden und hatte Wurzeln, die bis 1914 zurückreichten. Maßgeblich beteiligt war der Niederländer Henricus Sneevliet (Pseudonym: G. Maring), der als Komintern-Beauftragter auch die Gründung der KP Chinas begleitete. In der Komintern, der internationalen, zentralen Organisation kommunistischer Parteien (1919-1943), arbeiteten auch leitende PKI-Mitglieder wie Alimin, Musso und Tan Malaka, der 1948 die Murba-Partei als Alternative zur PKI gründete. ${ }^{19}$

In den 1920er und 1930er Jahren gab es eine Gründungswelle von KPs in den Kolonialgebieten, die zwar oft als Filialorganisation der KP der jeweiligen Kolonialmacht galten, aber eigene Sektionen innerhalb der Komintern bildeten. ${ }^{20}$ Die Parteien der Mutterländer wurden von der Komintern auf eine „besondere Verantwortung“ für die KPs ihrer Kolonien verpflichtet. ${ }^{21}$ Daher waren in ihrem Spitzengremium, dem Exekutivkomitee der Kommunistischen Internationale (EKKI), nur wenige Vertreter für außereuropäische Regionen vertreten: seit 1924 Manabendra Nath Roy für Indien und Tschin Du-liu für China sowie 1928

17 Zur Geschichte der PKI vgl. D. N. Aidit, Entstehung und Entwicklung der Kommunistischen Partei Indonesiens, 1920 bis 1955, Berlin 1956; Françoise Cayrac-Blanchard, Le Parti Communiste Indonésien, Paris 1973; Guy J. Pauker, The Rise and Fall of the Communist Party of Indonesia, in: Robert A. Scalapino (Hrsg.), The Communist Revolution in Asia: Tactics, Goals, and Achievements, Englewood Cliffs, N. J. ${ }^{2} 1969$, S. 274-307; Justus M. van der Kroef, The Communist Party of Indonesia. Its History, Program and Tactics, Vancouver 1965; George McTurnan Kahin, Nationalism and Revolution in Indonesia, Ithaca, N. Y. 1970, S. 70-87; Arnold Brackman, Indonesian Communism. A History, New York 1963; speziell zur Frühzeit: Ruth T. McVey, The Rise of Indonesian Communism, Ithaca, N. Y. 1965; J. Th. Petrus Blumberger, De nationalistische Beweging in Nederlandsch-Indië, Haarlem 1931.

${ }^{18}$ Die Bezeichnung „KP“ steht in diesem Beitrag aus pragmatischen Gründen stellvertretend für kommunistisch ausgerichtete Parteien, unabhängig von ihrer jeweiligen Selbstbezeichnung. Sie traten unter solch verschiedenen Bezeichnungen wie „Arbeiterpartei“, „Partei der Arbeit“, „Volkspartei“, „Sozialistische Partei“, „Volksavantgarde“ usw. auf.

19 Zur Mitarbeit der PKI vgl. Ragna Boden, Die Grenzen der Weltmacht. Sowjetische Indonesienpolitik von Stalin bis Brežnev, Stuttgart 2006, S.68f.; zur Komintern allgemein: Kevin McDermott/Jeremy Agnew, The Comintern: A History of International Communism from Lenin to Stalin, London 1996; Horst Schumacher, Die Kommunistische Internationale (1919-1943), Berlin [Ost] 1979; an Dokumenteneditionen sind hierzu zu nennen: Ja. S. Drabkin (Hrsg.), Komintern i ideja mirovoj revoljucii. Dokumenty [Die Komintern und die Idee der Weltrevolution], Moskau 1998; Hermann Weber, Die Kommunistische Internationale. Eine Dokumentation, Hannover 1966; zur Politik gegenüber Kolonien vgl. Komintern i Afrika. Dokumenty [Die Komintern und Afrika. Dokumente], zusammengestellt von Valentin Gorodnov, Sankt Petersburg 2003; Manuel Caballero, Latin America and the Comintern, 1919-1943, Cambridge 1986, mit einer Aufstellung der Teilnahme lateinamerikanischer Delegierter an den EKKI-Plenumssitzungen: S. $40 \mathrm{f}$.

${ }^{20}$ Vgl. Wolfgang Berner, Arabische Länder, in: C. D. Kernig, Die kommunistischen Parteien der Welt, Freiburg/Basel/Wien 1969, S.59-74, hier S. 65.

${ }^{21}$ Wolfgang Berner, Afrikapolitik, in: Osteuropa-Handbuch. Sowjetunion. Außenpolitik, 1955-1973, hrsg. von Werner Markert und Dietrich Geyer, Köln/Wien 1976, Bd. 2, S. 713-843, hier S. 718. 
Musso für Niederländisch-Indien. ${ }^{22}$ Während die Komintern im Jahr 192629 Parteien mit insgesamt 1,2 Millionen Mitgliedern repräsentierte, waren es 1935 bereits 61 Parteien mit 3,1 Millionen Mitgliedern. Ausschlaggebend für diesen starken Zuwachs waren wohl eher Radikalisierungstendenzen in der Arbeiterschaft als die eigene Propaganda- und Organisationsarbeit. ${ }^{23}$ Diese war vorwiegend auf Europa als nächstem Aktionsraum konzentriert. ${ }^{24}$ Zudem war die Mitgliedschaft sehr ungleich verteilt. Die Großregion Afrikas südlich der Sahara etwa war lediglich mit einer einzigen KP vertreten, der südafrikanischen. ${ }^{25}$

Nur in der Anfangsphase bis Mitte der 1920er Jahre fungierte die Komintern tatsächlich als Plattform zur Aushandlung politischer Vorstellungen und Vorgehensweisen, wo Kommunisten aus aller Welt die Gelegenheit hatten, ihre Visionen einer globalen Entwicklung zu diskutieren. ${ }^{26}$ Die hier gewonnenen Einsichten prägten im Folgenden die Vorgehensweise vieler KP-Führungen auch nach 1945, als die Befreiungsbewegungen ihren Höhepunkt erreichten. Im Wesentlichen war für die außereuropäischen Kommunisten die doppelte Aufgabe zu lösen, sowohl die europäische Fremdherrschaft abzuschütteln, als auch sich gegen einheimische Großgrundbesitzer und Regionalfürsten durchzusetzen. Strittig war seit dem zweiten Komintern-Kongress 1920 , wie dies zu bewerkstelligen sei. ${ }^{27}$ Gegen Lenins Vorschlag, dass die örtlichen Kommunisten zuerst und notfalls unter der Leitung des einheimischen Bürgertums und gemeinsam mit diesem die Kolonialmacht bekämpfen sollten, um danach einen sozialistischen Umsturz herbeizuführen, plädierten Sneevliet und M. N. Roy dafür, dass die Kommunisten direkt die Führung übernehmen sollten. Schon 1924 begann die „Bolschewisierung“ der Komintern, das heißt ihre Ausrichtung auf sowjetische Bedürfnisse. ${ }^{28}$ Die Verhaftungen und Vernichtungen im Zuge der sowjetischen „Säuberungen“ in den 1930er Jahren trafen schließlich auch ausländische Komintern-Mitarbeiter sowie Exil-Kommunisten. ${ }^{29}$

\footnotetext{
22 Vgl. die Zusammenstellung der Mitglieder bei Weber, Kommunistische Internationale, S. 371f. Roy wurde 1929 aus der Komintern ausgeschlossen.

2:3 Weber, Kommunistische Internationale, S. 21.

${ }^{24}$ Michael Weiner, Comintern in East Asia, 1919-39, in: McDermott/Agnew, Comintern, S. 158-196.

${ }_{25}$ Apollon Davidson/Irina Filatova, African History: A View from behind the Kremlin Wall, in: Maxim Matusevich (Hrsg.), Africa in Russia, Russia in Africa: Three Centuries of Encounters, Trenton, N. J. 2007, S. 111-131, hier S. 113.

${ }^{26}$ Vgl. etwa für die Außenseiterposition der KP Birmas: John H. Badgley, The Communist Parties of Burma, in: Robert A. Scalapino (Hrsg.), The Communist Revolution in Asia: Tactics, Goals, and Achievements, Englewood Cliffs, N. J. ${ }^{2} 1969$, S. 309-328.

${ }^{27}$ Der Zweite Kongreß der Kommunist[ischen] Internationale. Protokoll der Verhandlungen vom 19. Juli in Petrograd und vom 23. Juli bis 7. August 1920 in Moskau, Hamburg 1921. Zur sowjetischen Theorie über die Entwicklung der Kolonien in der Zeit 1920-1927 vgl. auch Xenia Joukoff Eudin/Robert C. North, Soviet Russia and the East, 1920-1927. A Documentary Survey, Stanford, Calif. 1957.

${ }^{28}$ Für die Bolschewisierung von oben und unten vgl. McDermott/Agnew, Comintern, S. 41-80; Schumacher, Kommunistische Internationale, S. 78-83; Weber, Kommunistische Internationale, S.19-21 („Stalinisierung“); speziell für Lateinamerika vgl. Wolfgang Berner, Die Sowjetunion und Lateinamerika, in: Osteuropa-Handbuch. Sowjetunion. Außenpolitik, Bd. 2, S. 844-878, hier S. 847; für die Machtverlagerung auf das EKKI vgl. Weber, Kommunistische Internationale, S. 18.

29. Christopher Andrew/Vasili Mitrokhin, The World Was Going Our Way. The KGB and the Battle for the Third World, New York 2005, S.504f. Anm. 8, nennen den Fall des Albert Nzula, des ersten schwarzen Generalsekretärs der KP Südafrikas, der im Gewahrsam des sowjetischen Geheimdienstes starb. Vgl. dazu auch, mit Dokumentenauszügen: Apollon Davidson u. a. (Hrsg.), South Africa and the Communist International: A Documentary History, Bd. 1, London/Portland, OR 2003, S. 17-21. Zum Terror in seinen Auswirkungen auf die Komintern insgesamt vgl. auch McDermott/Agnew, Comintern, S. $142-157$.
} 
Fraglich war auch, inwieweit die Komintern eine Basis für die Ausarbeitung von Kampfstrategien und die tatsächliche Vorbereitung von Revolutionen sein konnte. In der Mongolei hatten sich die Kommunisten 1921 mit Hilfe direkter sowjetischer Unterstützung durchgesetzt und drei Jahre später die Volksrepublik installiert. Dieser Erfolg war jedoch kein Anlass für Moskau, kommunistische Bewegungen andernorts vorbehaltlos zur Revolution zu ermutigen. Prägnante Beispiele für diese Politik der Zurückhaltung der Komintern waren der Umgang mit der KP Chinas und der PKI. Nachdem die chinesischen Kommunisten von der Komintern $1923 \mathrm{zu}$ einem Bündnis mit den Kuomintang (KMT) gedrängt worden waren, führten die von den KMT an ihnen verübten Massaker von April 1927 vor Augen, wie schlecht sie damit beraten waren. ${ }^{30}$ Nun setzte sich Mao innerhalb der KP Chinas durch und agierte zunehmend eigenständig, unabhängig von Moskau. ${ }^{31}$ In Indonesien scheiterten zu dieser Zeit die Ende 1926 - gegen den Willen der Komintern - begonnenen Aufstände der indonesischen Kommunisten auf Java und Bali. ${ }^{32}$ Beide Ereignisse zeigen, dass die Revolutionsstrategie der Komintern zwar um theoretische Fragen kreiste und Modelle aufzeigte, an denen sich viele Kommunisten aus Entwicklungsländern nach 1945 orientierten, sich aber in Fragen der konkreten Unterstützung von Befreiungsbewegungen sehr zurückhielt; sie zielte zunächst auf ein Bündnis mit bürgerlichen Kräften ab, bevor der Linksruck von 1928 eben solche taktischen Bestrebungen obsolet machte. Als Reaktion auf die weltpolitische Lage änderte sich die Ausrichtung der Komintern erneut, als seit 1934 ein breiteres antifaschistisches Bündnis angestrebt wurde. Dies galt zwar nicht mehr für den Fall Brasiliens im November 1935, doch zu dieser Zeit hatte die Komintern schon an Einfluss verloren. Das Scheitern des kommunistischen Aufstandes in Brasilien ebenfalls wegen fehlender Beteiligung aus der Bevölkerung - entmutigte dann weitere derartige Versuche. ${ }^{33}$ Vielversprechender schien die kooperative Taktik der kubanischen Kommunisten, zuerst mit den Nationalisten um Fulgencio Batista eine Volksfront zu bilden, um legalisiert (1938) und dann (1943) als erste lateinamerikanische KP an der Regierung beteiligt zu werden. ${ }^{31}$

\footnotetext{
30 Alexander Pantsov, The Bolsheviks and the Chinese Revolution, 1919-1927, Richmond, Surrey 2000; N. L. Mamaeva, Komintern i Gomin'dan, 1919-1929 [Die Komintern und die Kuomintang, 1919-1929], Moskau 1999; Dieter Heinzig, Sowjetische Militärberater bei der Kuomintang 1923-1927, Baden-Baden 1978.

31 Vgl. dazu Dieter Heinzig, Die Sowjetunion und das kommunistische China 1945-1950, Baden-Baden 1998, S. 9-17.

${ }^{32}$ Für die Komintern-Diskussionen vgl. „Discussion at Meeting of Indonesian Sub-secretariat [EKKI] July 29, 1926" (Rossijskij Gosudarstvennyj Archiv Social'no-Političeskoj Istorii [Russländisches Staatliches Archiv für Sozialpolitische Geschichte, im Folgenden: RGASPI]), f. 495, op. 154, d. 706, 1l. 74-87; Darsono [PKI-Mitglied] an das Exekutivkomitee der Komintern (EKKI), Moskau, Sekretariat für den Mittleren Osten, 16.11.1926 (RGASPI, f. 495, op. 154, d.303, Il. 1-2, hier 1. 1). Zu den Aufständen vgl. Harry J. Benda/Ruth J. [T.] McVey (Hrsg.), The Communist Uprisings of 1926-1927 in Indonesia: Key Documents, Ithaca, N. Y. 1960; van der Kroef, Communist Party, S. 3-44.

${ }^{33} \mathrm{Zu}$ Brasilien vgl. Boris Goldenberg, Kommunismus in Lateinamerika, Stuttgart 1971, S. 211-216, für die fehlende Unterstützung: S. 215f.

${ }^{34}$ Goldenberg, Kommunismus, S.91 und 293-305, und Hugh Thomas, Cuba or the Pursuit of Freedom, London 1971, S.706-715 und 733f.
} 


\section{Die kommunistischen Parteien in den Unabhängigkeitsbewegungen bis Mitte der 1950er Jahre}

Nach den vereinzelten Revolutionsversuchen der Kommunisten während der KominternZeit nahmen die Unabhängigkeitsbewegungen, die sich gegen die europäische Kolonialherrschaft und in Ost- und Südostasien auch gegen die japanische Besatzung richteten, nach dem Zweiten Weltkrieg neue Dimensionen an. ${ }^{35}$ Bis zur Mitte der 1950er Jahre erklärten vor allem süd- und südostasiatische und Staaten des Nahen Ostens ihre Unabhängigkeit, bevor 1960 in einem regelrechten Schub gleich 17 afrikanische Staaten folgten. Ein Teil der Zukunftshoffnungen der Komintern ging somit in Erfüllung. Nun mussten die kommunistischen Parteien es noch schaffen, sich an die Spitze der Bewegung zu setzen, um die neuen Staaten in ihrem Sinne auszugestalten.

Indonesien erklärte sich als einer der ersten Staaten nach dem Ende des Zweiten Weltkrieges unabhängig: Am 17. August 1945 riefen Sukarno und Mohammed Hatta die Republik aus. Beide gehörten weder der PKI an, noch hatten sie kommunistische Ambitionen, selbst wenn Sukarnos Weltbild neben nationalen und islamischen auch sozialistische Elemente umfasste. ${ }^{36}$ Damit stellte sich hier, wie bei den meisten KPs der unabhängig werdenden ehemaligen Kolonien die Frage, wie sich die Kommunisten im Unabhängigkeitskampf verhalten sollten.

Seit dem Zweiten Komintern-Kongress waren keine wesentlichen theoretischen Neuerungen für das Vorgehen der KPs der Kolonien hinzugekommen, so dass diese $1945 \mathrm{zu}-$ nächst auf sich gestellt waren. Ein gemeinsames Problem der KPs der Kolonien bestand neben ihrer Haltung zu den nichtkommunistischen Befreiungsbewegungen im eigenen Land in den Beziehungen zu den KPs ihrer Kolonialherren. Für beide Seiten gab es hier Schwierigkeiten mit den sich ausschließenden Loyalitäten. ${ }^{37}$ Insbesondere die französische KP sah sich nach dem Zweiten Weltkrieg im Konflikt zwischen ihrer Regierungsbeteiligung inklusive dem Erhalt der Kolonien einerseits und dem kommunistischen Grundsatz des Antiimperialismus andererseits. Der Zwiespalt stellte mittelbar auch ein Problem für die KPdSU dar. Die KP der Niederlande schwenkte offenbar von sich aus nach einer kurzen Phase, in der sie für die Beibehaltung der niederländischen Aufsicht über Indonesien plädiert hatte, auf die Linie um, der Republik Indonesien müsse die Unabhängigkeit gewährt werden. ${ }^{38}$ Somit war eine Unterstützung der Befreiungsbewegung im Archipel auch durch Moskau unproblematisch. ${ }^{39}$

35 Die Japaner hatten seit Dezember 1942 Indonesien und bis 1945 weite Teile Südostasiens besetzt und nach einer Phase der Konzessionen ein hartes Besatzungsregime eingeführt. Als sich die japanische Niederlage abzeichnete, trainierten die Besatzer in Indonesien noch einheimische Truppen, die zur Grundlage der Befreiungskämpfe auch gegen die Niederlande wurden.

${ }^{36}$ Bernhard Dahm, Sukarnos Kampf um Indonesiens Unabhängigkeit. Werdegang und Ideen eines asiatischen Nationalisten, Berlin 1966.

${ }^{37}$ Für Südostasien speziell vgl. Oliver E[dmund] Clubb, Jr., The United States and the Sino-Soviet Bloc in Southeast Asia, Washington, D. C. 1963, S. 14-16.

38 Für die Beibehaltung Indonesiens im Konnex mit den Niederlanden sprach sich Paul De Groot in seiner Rede vom 11.10.1945 aus: Internationaal Instituut voor Sociale Geschiedenis (Internationales Institut für Sozialgeschichte, Amsterdam), CPN, No.273, Bl.8; für das Abgehen von dieser Linie vgl. De Groots Rede vom November 1946: RGASPI, f. 17, op. 128, d. 161, 11. 41-49, bes. 43ob. 46 .

39 Für den Umgang der USA mit der Dekolonisierung in Südostasien vgl. auch Marc Frey, Dekolonisierung in Südostasien: die Vereinigten Staaten und die Auflösung der europäischen Kolonialreiche, München 2006. 
Diese Zurückhaltung in Fragen möglicher Revolutionen und Machtübernahmen durch die KPs in Kolonien und Entwicklungsländern war generell kennzeichnend für die StalinPhase. Den Sieg 1949 errangen die chinesischen Kommunisten weitgehend im Alleingang, während Stalin noch im August 1945 einen Bündnisvertrag mit den KMT geschlossen hatte. ${ }^{40}$ Weitere kommunistische Machtübernahmen gelangen partiell in Korea und Vietnam. Die staatliche Teilung beider Länder machte die Spaltung der Welt in zwei Lager für Asien ebenso deutlich wie das geteilte Deutschland für Europa. Aus Rücksicht auf die KP Frankreichs hielt die sowjetische Führung die vietnamesische Arbeiterpartei unter Ho Chi Minh bei der Genfer Friedenskonferenz 1954 mit ihren Forderungen zurück. Frankreich legte im Gegenzug ein Veto gegen die Gründung der Europäischen Verteidigungsgemeinschaft ein. ${ }^{41}$ Damit gab Moskau europäischen Belangen deutlichen Vorrang vor asiatischen, während die Volksrepublik China im gleichen Sinne, aber aus ganz anderen Motiven, auf die Viet Minh einwirkte. Peking wollte vor allem die USA aus der Pazifikregion fernhalten, um den eigenen Staat zu konsolidieren. ${ }^{42}$ Die KPs Vietnams und Koreas wiesen einige Gemeinsamkeiten in der Entwicklung auf, wie etwa die anfängliche Zersplitterung oder die zeitweilige Sozialisierung ihrer Gründer im Exil. Während aber die Viet Minh unter der Führung Ho Chi Minhs für die Bekämpfung der japanischen Besatzer mit den westlichen Alliierten kooperierten, kam Kim Il-Sung als Major der sowjetischen Streitkräfte nach Korea, um die japanische Herrschaft abzulösen. ${ }^{43}$

Im indonesischen Unabhängigkeitskampf, der 1950 mit der faktischen Lösung von den Niederlanden und der Aufnahme der RI in die UNO endete, kooperierte die PKI zunächst mit den indonesischen Regierungen und unterstützte selbst deren Abkommen mit den Niederländern. Da sie diese unpopulären Maßnahmen mittrug, wurde sie von der Bevölkerung kaum als eigenständige Kraft wahrgenommen. ${ }^{44}$ Dies änderte sich, als die PKI Ende 1948 einen Aufstandsversuch aus dem Militär unterstützte, der allerdings wie schon 1926 misslang. ${ }^{45}$ Zwar ordnete sich die Rebellion diesmal in eine Welle von kommunistischen

40 Heinzig, Die Sowjetunion und das kommunistische China, Kapitel 2.

41 Mari Olsen, Soviet-Vietnamese Relations and the Role of China, 1949-1964: Changing Alliances, New York 2006; Gerd Linde, Vietnam, in: Osteuropa-Handbuch. Sowjetunion. Außenpolitik, Bd.2, S.581-590, hier S.581. Mit der Verhinderung der Europäischen Verteidigungsgemeinschaft sollte auBerdem eine Wiederbewaffnung der Bundesrepublik Deutschland verhindert werden.

${ }^{42}$ Sergei N. Goncharov/John W. Lewis/Xue Litai, Uncertain Partners. Stalin, Mao, and the Korean War. Stanford, Calif. 1993; Shu Guang Zhang, Constructing „Peaceful Coexistence“: China's Diplomacy toward the Geneva and Bandung Conferences, 1954-55, in: Cold War History 7 (2007), S. 509-528, für die amerikanische Seite vgl. Yuen Foong Khong, Analogies at War: Korea, Munich, Dien Bien Phu, and the Vietnam Decisions of 1965, Princeton 1992.

${ }^{43}$ Für Vietnam vgl. Carlyle A. Thayer, Communist Party of Indochina, in: Haruhiro Fukui (Hrsg.), Political Parties of Asia and the Pacific, Bd. 2, Westpost, Conn./London 1985, S. 1102; dies., Vietnam Workers' Party, in: Fukui (Hrsg.), Political Parties, Bd. 2, S. 1181-1194; Marc Frey, Geschichte des Vietnamkriegs. Die Tragödie in Asien und das Ende des amerikanischen Traums, München ${ }^{4} 1999$, S. 16; für Korea vgl. Balázs Szalontai, Kim Il Sung in the Khrushchev Era: Soviet-DPRK Relations and the Roots of North Korean Despotism, 1953-1964, Washington, D. C. 2005.

44 Dieses Vorgehen kritisierte später der PKI-Generalsekretär, vgl. Aidit, Entstehung, S. 25.

${ }^{45} \mathrm{Zu}$ dieser sogenannten Madiun-Affäre vgl. Boden, Grenzen, Kapitel 1.3.2.2; aus der Perspektive der nachfolgenden PKI-Generation: D. N. Aidit, Konfrontasi Peristiwa Madiun 1948 - Peristiwa Sumatera 1956 [Die Konfrontation der Madiun-Ereignisse 1948 und der Ereignisse auf Sumatra 1956], Djakarta ${ }^{3}$ 1958; Aidit menggugat peristiwa Madiun [Aidit prangert die Madiun-Ereignisse an], Djakarta ${ }^{4} 1964$, auf Englisch unter folgendem Titel erschienen: Aidit accuses Madiun Affair (D. N. Aidit's Defence Plea at the Trial in the Jakarta Statc Court on February 24, 195.5), Djakarta 1955; Buku Putih tentang 
Aufständen in Asien ein: in Birma (März), Malaya (Juni) und in verschiedenen indischen Provinzen. Doch diese Rebellionen wurden sämtlich niedergeschlagen. ${ }^{46}$ Die PKI selbst wurde verboten, ihre Führung hingerichtet und der sogenannte Madiun-Aufstand innenpolitisch zu einem „Dolchstoß“ umgedeutet: Demnach sei die PKI der gegen die niederländischen Truppen kämpfenden indonesischen Unabhängigkeitsbewegung in den Rücken gefallen. ${ }^{47}$

Von westlichen Beobachtern wurden die hier geschilderten Aufstände zum Teil in Zusammenhang miteinander und mit der Formierung der Kommunisten in Entwicklungsländern gebracht. Ein Anhaltspunkt dafür war, dass sich 1948 unabhängig von der Etablierung kommunistischer Regime in Osteuropa die KPs der Entwicklungsländer in eigenen internationalen Versammlungen wie dem internationalen Jugendkongress (19.-25. Februar) in Indien organisierten. ${ }^{48}$ Die Bedeutung dieser Treffen als mögliche Auslöser der folgenden kommunistischen Aufstände in Südostasien ist unterschiedlich eingeschätzt worden. Zeitgenossen vermuteten hinter den Aufständen Pläne Moskaus, um zeitgleich mit Osteuropa auch Teile Asiens unter sowjetische Kontrolle zu zwingen ${ }^{49}$, oder sahen zumindest in der Rede Andrej Ždanovs von 1947 zusammen mit dem Jugendkongress in Indien eine Initialzündung für die Aufstände ${ }^{50}$. Soweit die sowjetischen Akten bisher zugänglich sind, lässt sich die These einer sowjetischen aktiven Beteiligung nicht belegen. Die Impulse scheinen vielmehr von den asiatischen KPs selbst ausgegangen zu sein..$^{51}$ In Indonesien fiel der Aufstand zwar mit der Rückkehr eines der führenden PKI-Mitglieder und langjährigen Exilanten in der Sowjetunion, Musso, zusammen. Aus seiner programmatischen Schrift für einen „Neuen Weg für die Republik Indonesien“52 lässt sich aber eine dezidierte Aufstandsanweisung aus Moskau nicht ablesen. Um die Bevölkerungen in den unabhängig werden-

Peristiwa Madiun [Weißbuch über die Madiun-Ereignisse], zusammengestellt und hrsg. vom Sekretariat Agitasi-Propaganda C. C. P. K. I., [Djakarta 1955].

46 Während die KPs Birmas und Malayas aus dem Untergrund heraus weiterkämpften, wurde auf den Philippinen im Oktober die von den Kommunisten geführte Widerstandsarmee der Huk mit Hilfe der von den USA ausgebildeten philippinischen Streitkräfte aufgerieben.

47 Vgl. hierzu van der Kroef, Communist Party, S. 44; Rex Mortimer, Indonesian Communism under Sukarno. Ideology and Politics, 1959-1965, Ithaca, N. Y. 1974, S.58.

${ }^{48}$ Für den Kongress vgl. Frank N. Trager, The Impact of Marxism, in: Ders. (Hrsg.), Marxism in Southeast Asia. A Study of Four Countries, Stanford, Calif. 1959, S. 240-299, bes. S. 263-273; George Benson, International Communism. Aims and Tactics outside the Sino-Soviet Bloc, Bd.2, o. O. 1961, Teil XXIV, S. 3; Dietmar Rothermund, Delhi, 15. August 1947. Das Ende kolonialer Herrschaft, München ${ }^{2} 1999$, Kapitel 1.

49 Für Indonesien vgl. Jeanne S. Mintz, Mohammed, Marx and Marhaen: the Roots of Indonesian Socialism, London/Dunmon 1965, S.92f.; Trager, Impact, S. 263-273; Benson, Communism, Bd. 2, Teil XXIV, S.3; dagegen Ann Swift, The Road to Madiun: The Indonesian Communist Uprisings of 1948, Ithaca, N. Y. 1989, S. 88.

${ }^{50} \mathrm{~J}$ [ack] H[enry] Brimmell, Communism in South East Asia. A Political Analysis, London/New York/ Toronto 1959, S. 263. Dagegen schätzten Swift, Road, S. 26-30, und Ruth T. McVey, The Calcutta Conference and the Southeast Asian Uprisings, Ithaca, N. Y. 1958, insbesondere den Einfluss der Konferenzen auf die $\mathrm{PKI}$ als gering ein.

51 Einen besonderen Einfluss Jugoslawiens auf die auf dem Jugendkongress anwesenden Delegierten sehen Gene D. Overstreet/Marshall Windmiller, Communism in India, Berkeley/Los Angeles 1959, S. 274.

52 Musso, Djalan Baru untuk Republik Indonesia (Rentjana Resolusi Polit-Biro untuk dimadjukan pada kongres ke-V dari Partai Komunis Indonesia. Disetudjui oleh konperensi PKI pada tanggal 26 dan 27 Agustus 1948) [Der neue Weg für die Republik Indonesien (Entwurf einer Resolution des Politbüros für den fünften Parteitag der PKI, angenommen von der PKI-Konferenz am 26.-27. August 1948)], Djakarta ${ }^{2} 1953$, abrufbar über: http://www.geocities.com/edicahy/marxist/pki/djalan-baru.html. 
den Staaten zu lenken, hatte die UdSSR zu dieser Zeit kaum die Möglichkeiten und Institutionen.

Das Problem stellte sich schon damit, dass in der Nachkriegsepoche kein Pendant zur 1943 aufgelösten Komintern existierte, welches die außereuropäischen KPs einbezogen hätte. Passend zu seiner im Wesentlichen auf Europa konzentrierten Außenpolitik organisierte Stalin 1947 die Kominform als supranationale kommunistische Organisation mit europäischer Ausrichtung. ${ }^{.33}$ Äußerungen Stalins zu einer Aufteilung der Einflusssphären zwischen der UdSSR und China, nach denen Moskau sich um die europäischen und Peking um die KPs der Kolonien und Entwicklungsländer kümmern sollte, sind als rein taktisches Manöver zu verstehen, um Mao zufriedenzustellen. ${ }^{54}$

Die Verbindung zu den KPs der Kolonien und Entwicklungsländer funktionierte wegen der zunehmenden Differenzen zwischen Moskau und Peking nur noch eingeschränkt und mit wachsenden Verlusten. Die gemeinsamen Foren in Form der kommunistischen Weltkonferenzen, die in den Jahren 1957, 1960, 1969 abgehalten wurden ${ }^{55}$, litten darunter ebenso wie die Feier zum 70. Jubiläum der Oktoberrevolution in Moskau, an der auch Vertreter von 40 Parteien und Bewegungen aus der Dritten Welt teilnahmen ${ }^{56}$. Das kommunistische Netzwerk reichte damit nicht mehr an das Vorkriegsniveau heran.

Einen Ersatz mit reduzierter Besetzung bildeten die KPdSU-Parteitage, zu denen die KPFührungen aus aller Welt geladen waren, um sich über die sowjetische Linie zu informieren. Aus der Präsenz ihrer Abgesandten und der Reihenfolge ihrer Nennung bei der Begrüßung ließ sich jeweils ihre Nähe zur KPdSU ablesen.$^{57}$ Diese Anlässe boten Gelegenheit zum internationalen Austausch; ansonsten hielt Moskau die Beziehungen insbesondere zu den nichtregierenden, vorwiegend außereuropäischen KPs über die Internationale Abteilung (IA) des Zentralkomitees der VKP(b)/KPdSU aufrecht, die fast während der gesamten Zeit ihres Bestehens von Boris Ponomarev geleitet wurde (1955-1986). ${ }^{58}$ Die Internationale Abteilung gliederte sich in funktionale und geographische Sektoren. Sie wechselten

53 Editionen der Sitzungsprotokolle: Soveščanija Kominforma, 1947, 1948, 1949. Dokumenty i materialy [Die Komintern-Konferenzen, 1947, 1948, 1949. Dokumente und Materialien], Moskau 1998; Giuliano Procacci (Hrsg.), The Cominform: Minutes of the Three Conferences 1947/1948/1949, Mailand 1994.

54 Vgl. dazu Grant M. Adibekov, Das Kominform und Stalins Neuordnung Europas, hrsg. von Bernhard H. Bayerlein und Jürgen Mothes, in Verbindung mit Olaf Kirchner, Frankfurt a. M. 2002, S. 121-126; Heinzig, Die Sowjetunion und das kommunistische China, S. 358-361.

55 Allerdings waren seit 1960 wegen des sino-sowjetischen Konfliktes nicht mehr alle KP-Führungen vertreten. Kennzeichnend für die internationalen Konferenzen der kommunistischen und Arbeiterparteien war die Frage nach einer besonderen sowjetischen Stellung innerhalb des sozialistischen Weltsystems. Hatte Mao 1957 noch auf einer Führungsposition der KPdSU bestanden, so betonte das Schlusspapier der Konferenz von 1960 die Gleichberechtigung und Unabhängigkeit der KP. Vgl. Heinz Brahm, Der sowjetisch-chinesische Konflikt, in: Osteuropa-Handbuch. Sowjetunion. Außenpolitik, Bd. 2, S. 469-536, hier S. 474 und 489. Vgl. dazu auch unten, S. 133.

${ }^{56}$ Heinz Timmermann, Die KPdSU und das internationale kommunistische Parteiensystem. Paradigmenwechsel in Moskau, Köln 1989 (Berichte des Bundesinstituts für ostwissenschaftliche und internationale Studien [BBIOSt], H. 13, 1989), S.6f.

57 Robert W. Kitrinos, International Department of the CPSU, in: Problems of Communism (PoC) 33 (1984), H.5, S. 47-75, hier S. 69 Anm.8.

58 Zur Internationalen Abteilung vgl. Kitrinos, International Department; Boris Meissner, Das außenund sicherheitspolitische Entscheidungssystem der Sowjetunion, in: Aus Politik und Zeitgeschichte B 43/1983, S.31-45; Leonard Schapiro, The International Department of the CPSU: Key to Soviet Policy, in: International Journal 32 (1976/77), S. 41-55. 
zwar im Laufe der Jahre in ihrer Zusammensetzung, im Wesentlichen blieb es für die Entwicklungsländer aber bei den Großregionen Naher Osten/Arabische Staaten, Südasien, Südostasien, Lateinamerika, und Afrika südlich der Sahara. ${ }^{59}$ Die IA war weiterhin das wichtigste sowjetische Verbindungsorgan zu den KPs bis zum Ende der UdSSR. Hier arbeiteten auch Genossen aus den Entwicklungsländern wie Indonesien, die unter anderem Übersetzungsdienste leisteten. ${ }^{60}$

\section{Die Dekolonisierungswelle der 1950er/60er Jahre}

Während Peking in den Unabhängigkeitsbewegungen potentielle Partner für eine Einflussnahme sah, hatte Moskau sichtlich Mühe, ein Konzept für den Umgang mit außereuropäischen KPs zu entwickeln. Soweit die Verbindungen bisher erforscht sind, war Stalin an den Beziehungen zu außereuropäischen KPs weniger interessiert. ${ }^{61}$ Chruščev dagegen unternahm zwar etliche Anläufe, um Beziehungen mit Entwicklungsländern in Form der Aufnahme diplomatischer Beziehungen, Wirtschafts- und Militärhilfe anzuknüpfen; er stellte aber die Verbindungen mit den KPs dieser Staaten dafür hintan, wenn sie die Staatsführung des Entwicklungslandes irritierten, was sich besonders deutlich in Ägypten und Indonesien zeigte. ${ }^{62}$ Teilweise - so in den genannten Ländern - wurden die KPs vor allem dazu genutzt, um die Beziehungen auf staatlicher Ebene zu verbessern. Doch war dies zumindest in der Inselrepublik kein einseitiges Vorgehen der sozialistischen Supermacht; auch der PKI-Generalsekretär Aidit sah durchaus Vorteile in einer bilateralen Annäherung auf Staatsebene und riet zu entsprechenden Aktivitäten in Moskau, die nicht nur die Verbindungen zur PKI, sondern auch zu den anderen großen indonesischen Parteien umfassen sollten. ${ }^{63}$

Insgesamt unterschieden sich die Ausgangsbedingungen für die Beziehungen zu den KPs in den Ländern der Dritten Welt gewaltig. Der Einfluss einer Partei hing ganz wesentlich von der Machtstellung im eigenen Land ab: ob sie die politisch beherrschende Kraft war wie in Nordkorea, Kuba usw., an der Regierung beteiligt wie in den 1960ern in Indonesien oder in Parlamenten vertreten wie in Indien, ob sie wenigstens legal war oder aber illegal wie in den meisten arabischen Staaten. In jedem Fall spielte auch die Frage der Loyalität der jeweiligen KP zu Moskau eine Rolle.

Nach ihrem zeitweiligen Verbot als Reaktion auf den gescheiterten Aufstand 1948 gelang es der PKI diesmal recht schnell, sich wieder zu erholen. Unter der neuen Führung Aidits beteiligte sich die Partei an den ersten indonesischen Parlamentswahlen 1955 und wurde mit 16 Prozent viertstärkste Kraft nach den Nationalisten (PNI) und den Islam-Parteien. ${ }^{64}$ Damit schaffte es die PKI, sich fest in der politischen Landschaft Indonesiens zu etablieren. Sie baute ihre Organisationen aus, schuf Einrichtungen der Gewerkschaft, für

${ }^{59}$ Kitrinos, International Department, S. 68 (Stand ca. 1983/84).

${ }^{60}$ Vgl. RGASPI, f. 17, op. 128 , d. 633 , ll. 40-48.

61 Vgl. am Beispiel Indonesien: Boden, Grenzen, Kapitel 1.3.

${ }^{62}$ Für Ägypten vgl. Alvin Z. Rubinstein, Red Star on the Nile. The Soviet-Egyptian Influence Relationship since the June War, Princeton, N. J. 1977, S. $21 \mathrm{f}$; für Indonesien Ragna Boden, Cold War Economics: Soviet Aid to Indonesia, in: Journal of Cold War Studies 10 (2008), H. 4, S. 110-128.

${ }^{63}$ Gespräch des stellvertretenden Außenministers Kuznecov mit Aidit, 6.5.1956 (AVP RF, f.091, 12-10-2, 11. 14-17).

${ }^{64}$ Herbert Feith, The Indonesian Elections of 1955, Ithaca, N. Y. ${ }^{2} 1971$; Ruth T. McVey, Nationalism, Revolution, and Organization in Indonesian Communism, in: Daniel S. Lev/Ruth T. McVey (Hrsg.), Making Indonesia, Ithaca, N. Y. 1996, S.96-117, hier S. 98. 
die Jugend, Frauen und Künstler ${ }^{65}$ Dadurch und durch intensive Propagandaarbeit stieg sie bis 1965 zur weltweit mitgliederstärksten nichtregierenden KP auf; sie war mit bis zu 3,5 Millionen Mitgliedern und 20 Millionen organisierten Anhängern (so die eigenen Angaben ${ }^{66}$ nach der KPdSU und der KP Chinas die drittgrößte im globalen Maßstab und in ihrer Bedeutung kaum zu überschätzen. Die PKI formierte sich damit als Massen- statt als Kaderpartei ${ }^{67}$ und versammelte etwa 3,5 Prozent der Bevölkerung in ihren Reihen. ${ }^{68}$ Hinsichtlich der sozialen Zusammensetzung überwog in ihrer Funktionselite die Mittelschicht mit mittlerer, selten höherer Bildung. Nach einer Zählung Anfang der 1960er Jahre gehörten 28 Prozent der Parteimitglieder zum Proletariat und 72 Prozent zum Bürgertum oder waren Bauern. ${ }^{69}$ Damit lag der Anteil der Arbeiterschaft nicht sehr viel niedriger als zur gleichen Zeit in der KPdSU, wo er 34 Prozent betrug, gegenüber 17,5 Prozent Bauern. ${ }^{70}$ Diese Tendenz war insofern charakteristisch für die KPs der Entwicklungsländer, als sich aufgrund des meist niedrigen Industrialisierungsgrades kaum ein Arbeiterproletariat entwickeln konnte und die Kolonialmächte nur wenigen Einheimischen höhere Bildungsmöglichkeiten eröffnet hatten. Daher zielten viele außereuropäische KPs darauf ab, zunächst die Modernisierung ihres Landes voranzutreiben. ${ }^{71}$ Ihre Führungen dachten viel eher als das Moskauer Zentrum in der Kategorie von multiplen Formen der Moderne. ${ }^{72}$ Diese Absicht war eng verbunden mit dem Streben nach einem eigenständigen Weg zum Sozialismus, was die PKI jedoch keineswegs als Zielkonflikt empfand.

Kennzeichnend für die PKI wie für viele KPs der Entwicklungsländer war zudem eine ausgeprägte Abneigung gegen Fremdvereinnahmung. Als Vertreterin einer postkolonialen Nation bewahrte sie sich lange eine Eigenständigkeit innerhalb der kommunistischen Weltbewegung, die zur Ausbildung eines nach ihrem Generalsekretär benannten „Sozialismus à la Aidit" führte. ${ }^{73}$ Darin ähnelte sie den Bruderparteien in Kuba, Korea und Vietnam, auch wenn die PKI keine Ausstrahlung auf andere Regionen hatte. ${ }^{74}$ Diese Unabhän-

${ }^{65}$ Vgl. Justus M. van der Kroef, Indonesian Communism's Cultural Offensive, in: Australian Outlook (April 1965), S. 40-61; Cayrac-Blanchard, Parti, S. 80-84.

66 Für das Jahr 1963 gab der PKI-Generalsekretär Aidit gegenüber deutschen Genossen die Zahl von 3,5 Millionen an, von denen aber offiziell nur 2,5 Millionen genannt würden, vgl. „Aidits Ausführungen am 10.8. 1963 gegenüber Gen. Hager“ (SAPMO-BArch, DY/30/IV A 2/20, 667, o. P., 1. Bl.).

${ }_{67}$ McVey, Nationalism, S. 101.

68 Zum Vergleich: In Vietnam stellten die 570000 Mitglieder der Vietnamesischen Arbeiterpartei im Jahre 19633 Prozent der Bevölkerung. Vgl. dazu John C. Donnell/Melvin Gurtov, North Vietnam: Left of Moscow, Right of Peking, in: Robert A. Scalapino (Hrsg.), The Communist Revolution in Asia: Tactics, Goals, and Achievements, Englewood Cliffs, N. J. ${ }^{21969, ~ S . ~ 152-183, ~ h i e r ~ S . ~ 152 . ~ Z u ~ w e i t e r e n ~}$ Vergleichen siehe unten, S. 140.

69 McVey, Nationalism, S. 100 Anm. 4.

70 Vgl. Manfred Hildermeier, Geschichte der Sowjetunion 1917-1991: Entstehung und Niedergang des ersten sozialistischen Staates, München 1998, S. 1183.

${ }^{71}$ McVey, Nationalism, S. 107; für die Mongolei vgl. Odd Arne Westad, The Global Cold War: Third World Interventions and the Making of Our Times, Cambridge 2005, S.51.

${ }^{72}$ Vgl. auch Johann P. Arnason, Communism and Modernity, in: Shmuel N. Eisenstadt (Hrsg.), Multiple Modernities, New Brunswick/London 2002, S.61-90, bes. S. 79-82.

${ }^{73}$ Peter Edman, „Communism à la Aidit": the Indonesian Communist Party under D. N. Aidit, 1950-1965, Townsville 1987; Justus M. van der Kroef, Indonesian Communism under Aidit, in: PoC 7 (1958), H. 6, S. 15-23.

${ }^{74}$ Für die Ausstrahlung Vietnams in Indochina vgl. Renate Strassner, Der Kambodscha-Konflikt von 1986-1990 unter besonderer Berücksichtigung der Rolle Vietnams, Münster/Hamburg 1991, S. 21; für Korea: Chong-Sik Lee, Stalinism in the East: Communism in North Korea, in: Robert A. Scalapino (Hrsg.), The Communist Revolution in Asia: Tactics, Goals, and Achievements, Englewood Cliffs, N. J. '1969, S. 120-150, hier S. 129f. 
gigkeit war unabdingbar für den innenpolitischen Erfolg der Partei. Hatten Rebellionen mit kommunistischer Beteiligung reflexartig die Furcht vor sowjetischer Einflussnahme geweckt, so versuchte die PKI seitdem, sich mit einem betont indonesischen Programm von solchem Verdacht abzusetzen. Als asiatischer Partei fiel es ihr weniger schwer, die Distanz zu Moskau plausibel zu machen. Anders verhielt es sich mit der Beziehung zur KP Chinas. Seit ihrem Machtantritt 1949 übernahm jene - mit Wissen und Billigung Stalins die Verbindung zwischen der VKP(b)/KPdSU und einigen asiatischen KPs. ${ }^{75}$ Nachweisbar ist diese Verbindung auch für die PKI, in der Stalin sich allein aufgrund der chinesischen Informationen zur Lage in Indonesien zum PKI-Programm äußerte. ${ }^{76}$ Damit waren die Rollen klar verteilt: Die chinesischen Kommunisten lieferten die nötigen Informationen, während Stalin sich die Bewertung und Beratung vorbehielt. Dies war jedoch nur so lange möglich, wie Einvernehmen zwischen Moskau und Peking herrschte.

Mit dem offenen Bruch zwischen der UdSSR und der Volksrepublik China 1961 entstand eine Situation, in der sich jede KP zwischen zwei sozialistischen Zentren entscheiden musste. ${ }^{77}$ Viele hatten bis dahin versucht, wie die PKI zwischen den Kontrahenten zu vermitteln oder wenigstens zwischen beiden Lagern laviert, wie die vietnamesischen und die koreanischen Kommunisten, bis eine Entscheidung unausweichlich war. Weltweit, so auch bei den KPs der Entwicklungsländer, zeitigte dies unterschiedliche Resultate: entweder die KP folgte in toto einer Observanz wie die traditionell pro-sowjetische Mongolei ${ }^{78}$ oder die tendenziell zu Moskau neigenden Kubaner. Letztere neigten jedoch ebenso wie viele der afrikanischen marxistisch-leninistischen Parteien ${ }^{79}$ sehr zur Eigenständigkeit. Pro-chinesisch waren dagegen die meisten der südostasiatischen KPs ausgerichtet, wie die in Birma, Thailand, Malaya. Konnten sich die Parteien nicht auf eine Gefolgschaft einigen, konnte es zu Spaltungen kommen, wie auf den Philippinen und auf Sri Lanka geschehen. ${ }^{80}$ Manche KPs waren auch schon vorher in mehrere Flügel zerfallen wie in Birma seit 1946 oder Indien. Für die internationale kommunistische Bewegung bedeutete dies, dass die nach außen hin demonstrierte Einheitlichkeit, die bereits früh (1948) durch die jugoslawische Eigenständigkeit durchbrochen worden war, nun endgültig verloren war. Auch Fragen der geographischen Lage spielten eine Rolle. So entwickelte das maoistische Modell seine Anziehungskraft vor allem in Asien, wo es besser auf die dortigen Verhältnisse übertragbar schien als das der Bolschewiki. ${ }^{81}$ Allerdings war die geographische Nähe nicht immer ein Anlehnungskriterium, sondern sie wirkte wegen der potentiellen Bedrohung auch absto-

75 Liu Shaoqui hatte sogar verkündet, dass Maos Weg zur Macht für alle Völker Asiens und der unterentwickelten Welt richtungsweisend sei. Vgl. Stuart R. Schram, The Political Thought of Mao Tse-tung, New York/Washington/London '1969, S. 111.

$76 \mathrm{Vgl}$. Boden, Grenzen, Kapitel 1.3.

77 Dazu jetzt Lorenz Lüthi, The Sino-Soviet Split: The Cold War in the Communist World, Princeton 2008.

78 Sergey S. Rachenko, The Soviet's Best Friend in Asia: The Mongolian Dimension of the Sino-Soviet Split, Washington 2003 (CWIHP Working Paper No.42); M. T. Haggard, Mongolia: The First Communist State in Asia, in: Robert A. Scalapino (Hrsg.), The Communist Revolution in Asia: Tactics, Goals, and Achievements, Englewood Cliffs, N. J. ${ }^{21969, ~ S . ~ 85-119, ~ h i e r ~ S . ~} 96 \mathrm{f}$.

79 Fritz. Schatten, Schwarzafrika, in: C. D. Kernig (Hrsg.), Die kommunistischen Parteien der Welt, Freiburg/Basel/Wien 1969, S. 447-457, hier S.448.

${ }^{80}$ Kevin Devlin, Die kommunistischen Parteien in Asien zwischen Moskau und Peking, in: Joachim Glaubitz/Dieter Heinzig (Hrsg.), Die Sowjetunion und Asien in den 1980er Jahren, Baden-Baden 1988, S. 99-108, hier S. 100 und 107f.

81 Vgl. dazu auch Robert A. Scalapino, Südostasien, in: Kernig (Hrsg.), Die Kommunistischen Parteien der Welt, S. 490-495, hier S. 495. 
Bend. Während etwa die europäischen KPs Albaniens und bedingt auch Rumäniens China zuneigten, verhielt sich die mongolische KP loyal zu Moskau.

Die Spaltung der kommunistischen Weltbewegung hatte auch zur Folge, dass sich einige KPs nun (noch) mehr auf ihre Eigenständigkeit besannen - so die KP Indiens ${ }^{82}$ - oder sich der einflussreichsten KP ihres Kontinents zuwandten, wie Venezuelas KP den kubanischen Genossen, mit denen es allerdings auch zu Auseinandersetzungen kam. ${ }^{83}$ Für die afrikanischen KPs fiel der sino-sowjetische Streit in die erste Phase der Dekolonisierung, die gleichzeitig eine Orientierungsphase war. ${ }^{84}$

Der sino-sowjetische Bruch erhielt für die KPs der Entwicklungsländer noch zusätzliches Gewicht dadurch, dass China gezielt auf die gemeinsame Identität der außereuropäischen Völker abhob. So bemühte sich Peking mit Erfolg, die Vertreter der UdSSR bei den in dieser Zeit beginnenden afro-asiatischen Kongressen als vorwiegend europäische Macht außen vor zu lassen. ${ }^{85}$ Eng mit diesen Kongressen verbunden war die Blockfreienbewegung. In ihrer Grundidee, dass sich die in ihr vernetzten Staaten keinem der Machtblöcke des Ost-West-Konfliktes anschließen und eine Distanz gegenüber den kapitalistischen wie den sozialistischen Staaten wahren sollten, berührte sie Kerninteressen der KP. Auch bei der Bewegung der Blockfreien spielte Indonesien eine wesentliche Rolle. Zur BandungKonferenz im April 1955 wurde die UdSSR als Teilnehmerin mit dem Hinweis auf ihren vorwiegend europäischen Charakter nicht eingeladen. Die Ausschlusstendenzen von diesen Konferenzen verschärften sich in den Folgejahren und brachten Moskau dazu, ein anderes afro-asiatisches Gremium aktiv zu unterstützen, die Afro-Asian People's Solidarity Organization, die zunächst sowjetfreundlich ausgerichtet war, bevor die Chinesen auch hier als Konkurrenz auftraten. ${ }^{86}$

Überrollt von der faktischen Entwicklung der Dekolonisierung, versuchte die sowjetische Führung unter Chruščev, mit den Ereignissen auch auf theoretischer Ebene Schritt zu halten. So sollte unter anderem die Diskrepanz zwischen den national gesinnten Kräften der neuen Staaten und den selten im Prozess der Unabhängigkeitskämpfe führend auftretenden KPs vermindert werden. Daraus entstanden Konstrukte wie das der „Staaten der nationalen Demokratie“, das als besondere Etappe der Entwicklungsländer auf dem Weg zum Sozialismus galt und für dessen Errichtung eine „fortschrittliche“ (d.h. kommunistische) Partei nötig war. ${ }^{87}$ Indonesien wurde zusammen mit Ägypten, Algerien, Birma, Ghana, Kongo (Brazzaville), Kuba und Mali als ein solcher Staat der nationalen Demokra-

${ }^{82}$ Indira Rothermund, Die Spaltung der Kommunistischen Partei Indiens. Ursachen und Folgen, Wiesbaden 1969, S.92.

${ }^{83}$ Robert J. Alexander, The Communist Party of Venezuela, Stanford, Calif. 1969, S. $201-204$.

${ }^{44}$ Vgl. dazu A. M. Babu, African Socialism or Socialist Africa?, London 1981, S. 101.

85 Vgl. hierzu Brahm, Konflikt, S. 513.

86 Vgl. dazu Roy Allison, The Soviet Union and the Strategy of Non-alignment in the Third World, Cambridge 1988, S. 29f.; Paul F. Power, The People's Solidarity Movement: Evolution and Continuity, in: Mizan USSR, China, Africa, Asia, 9 (1967), H. 1, S. 10-22; D. G. Toeppel, Kommunistische Weltorganisationen, in: C. D. Kernig (Hrsg.), Die kommunistischen Parteien der Welt, Freiburg/Basel/Wien 1969, Sp. 37-50, bes. Sp.49; für den sowjetischen Standpunkt: Kenesbaj Kožachmetovič Kožachmetov, Dviženie solidarnosti narodov Avii i Afriki v bor'be s kolonializmom i imperializmom (1955-1960gg.) [Die Solidaritätsbewegung der Völker Asiens und Afrikas im Kampf gegen Kolonialismus und Imperialismus], Alma Ata 1983; für den chinesischen Einfluss Omar Ali Amer, China and the Afro-Asian Peoples' Solidarity Organization 1958-1967, Diss. Genf 1972, bes. Kapitel IV-VI.

s7 Die Moskauer Frklärung von 1960, in: Ost-Probleme 13 (1961), H.3, S.66-83, bes. S. $77 f$. 
tie deklariert. Obwohl die PKI diese Definition zunächst ablehnte, gestand sie sie später zu. ${ }^{\text {s. }}$

Die Moskauer Strategie spiegelte die Situation wider, dass kaum eine Regierung der neuen Staaten die jeweilige KP beteiligte und in vielen Ländern nicht einmal eine solche Partei vorhanden war. ${ }^{89}$ Selbst sozialrevolutionäre und von Moskau favorisierte Führungen in afrikanischen Ländern waren selten bereit, eine institutionalisierte KP zu dulden, noch weniger, sie an der Regierung zu beteiligen. ${ }^{90}$ Moskau ging sogar so weit, solche KPs zur Selbstauflösung zu drängen, die die außenpolitischen Beziehungen der UdSSR mit den Staatsführungen störten; so geschehen in Nassers Ägypten. ${ }^{91}$ Die sowjetische Führung war zeitweise bereit, jede Form von Umgang mit Kommunisten zu tolerieren, bis hin zu Repressalien wie in Ägypten und dem Irak, wo Kommunisten 1961 bzw. 1978 verfolgt wurden. ${ }^{92}$ Nicht selten half der sowjetische Geheimdienst KGB den Einparteien-Regimes, die Opposition zu unterdrücken. ${ }^{93}$

Um der Vielfältigkeit der sozialistischen Weltbewegung Rechnung zu tragen, wurde 1964 die Kategorie der Staaten der „revolutionären Demokratie“ eingeführt. Als Revolutionäre Demokratien konnten sich solche Staaten qualifizieren, die „fest mit den Massen verbunden [waren], konkrete Methoden und Formen des Übergangs zum sozialistischen Weg“ suchten. ${ }^{94}$ Damit fielen Staaten wie Indonesien, wo dieser Prozess noch nicht so weit gediehen war, noch nicht unter diese Kategorie.

\section{Die Brežnev-Epoche}

Eine Zäsur in der Moskauer Politik gegenüber den Entwicklungsländern ergab sich Mitte der 1960er Jahre. Sie war bedingt einerseits durch den Wechsel in der sowjetischen Führung, andererseits durch die gewaltsame Ablösung etlicher von Moskau unterstützter Regierungen. In Indonesien verlor die KP ihren wichtigsten Halt in Gestalt des Präsidenten Sukarno und die Auseinandersetzungen fanden ein blutiges Ende. Seit März 1966 war sie offiziell verboten, und im Juli desselben Jahres wurde ein Gesetz über das Verbot der Verbreitung des Kommunismus und des Marxismus-Leninismus verabschiedet. ${ }^{95}$ Die letzten Kader der PKI, die nicht auf der Stelle ermordet, sondern für öffentliche Prozesse aufgespart worden waren ${ }^{\% ;}$, wurden zum Tode verurteilt und 1968 trotz Protesten auch aus der UdSSR hingerichtet. Von diesem Rückschlag erholte sich die PKI, die bis dahin schon zwei Verfolgungen erlebt hatte, nicht mehr. Stattdessen bildeten sich Exilgruppen vornehmlich

\footnotetext{
${ }^{8 *}$ Zur Ablehnung vgl. Aktennotiz Radde an die ZK-Abteilung Außenpolitik und Internationale Verbindungen, 19.4.1961 [Gespräch mit den PKI-Mitgliedern Naibaho, Sutjipto, Slamet] (SAPMOBArch, DY 30/IV 2/20, 324, o. P.: 2 Bl., hier 2. Bl.). Zur Billigung vgl. McVey, The Rise of Indonesian Communism, S.373 Anm. 25.

89) Siehe dazu unten, S. 140.

90 Berner, Afrikapolitik, S. $740 \mathrm{f}$.

91 Berner, Arabische Länder, S. 67.

92 Charles B. McLane, Soviet-Middle East Relations, London 1973, Bd. 1, S. 55; Berner, Arabische Länder, S. 65.

93 Andrew/Mitrokhin, World, S. 487.

94 K[aren] N. Brutenc, Sovremennyj ètap nacional'no-osvoboditel'nogo dviženija [Die aktuelle Etappe der nationalen Befreiungsbewegung], in: Kommunist 17 (1964), S. 23-34, bes. S. 30.

95 A[leksandr] Belen'kij/B. Il'ičev, Nekotorye uroki sobytij Indonezii [Einige Lehren aus den Ereignissen in Indonesien], in: Kommunist 15 (1968), S. 110-123, hier S. 116.

96 Cribb/Brown, Modern Indonesia, S. 106.
} 
in der UdSSR und China, die sich gegenseitig die Verantwortung für das Desaster von 1965 zuschoben ${ }^{97}$ Die Maoisten attackierten in ihren Schriften die „modernen Chruščev'schen Revisionisten“ und folgerten, nur ein bewaffneter Kampf könne nun der PKI zur Macht verhelfen. Dagegen bevorzugten die in der Sowjetunion Exilierten eine Verhandlungsstrategie, für die jedoch jegliche Ansprechpartner in Indonesien fehlten. Ohnehin erlangte keine der Gruppierungen Einfluss in Indonesien. Auch in Ghana, Mali, Guinea und Algerien mussten die sowjetfreundlichen Führungen Mitte und Ende der 1960er Jahre Militärregierungen weichen. Somit war Chruščevs Taktik des Umgangs mit den KPs der Entwicklungsländer offensichtlich gescheitert.

Die neue Führung unter Leonid Brežnev und Aleksej Kosygin ersetzte daher das Chruščev'sche Prinzip der Wirtschafts- und Militärhilfe, die schon bei geringer Übereinstimmung der politischen Ziele angesetzt und kaum Rücksicht auf die örtlichen KPs genommen hatte, durch eine aktive Unterstützung revolutionärer Bewegungen und einen deutlichen Schwerpunkt auf der militärischen Hilfe für sozialistische Gruppierungen und KPs. Allerdings lässt sich eine klare Linie auch in dieser Zeit nur in Ansätzen feststellen. Das neue, eher unsystematisch eingesetzte Instrument der Freundschaftsverträge, das für diese Zeit charakteristisch war, ist ein Indikator dafür. ${ }^{98}$ Zumindest erhielten Militärregime, in denen die KP verfolgt wurde, nun, selbst wenn Interesse von ihrer Seite bekundet wurde, selten Lieferungen. Damit konnte der neue indonesische Machthaber Suharto nicht automatisch, wie etwa der ägyptische Präsident Sadat nach dem Tode Nassers 1970, auf eine Perpetuierung der Unterstützung hoffen. Mit dem neuen ägyptischen Staatschef schloss die UdSSR 1971 sogar einen Freundschaftsvertrag, der allerdings nicht lange hielt: Ein Jahr später wurden die sowjetischen Militärberater aus Ägypten ausgewiesen.

Die öffentliche Beteuerung eines sowjetischen Experten von 1967, Kommunismus könne nicht exportiert werden ${ }^{99}$, schien sich zu bewahrheiten. Zumindest für den Bereich der sowjetischen Variante war die einfache Umsetzung fraglich, denn der Sozialismus erlebte nicht nur länder-, sondern auch regionalspezifische Ausprägungen wie den Afromarxismus. ${ }^{100}$ In einer nachholenden Debatte versuchten die Marxisten der Entwicklungsländer, die Klassiker des Kommunismus vom europäischen Kontext zu lösen und für die spezifische Gesellschafts- und wirtschaftliche Situation ihres Landes oder ihrer Region zu adaptieren. Ihr Argument war, dass die Idee des Sozialismus im 19.Jahrhundert aus europäischer Perspektive entwickelt und in dieser Form nicht auf die postkolonialen Staaten anwendbar sei. In einer prägnanten Formulierung brachte es Senegals Präsident Leopold Senghor auf den Punkt: „Marx war kein Afrikaner." "Vielmehr war man auf der Suche nach den allgemeingültigen Gesetzen des Sozialismus, entkleidet von europaspezifischen Handlungsanleitungen. So wurde gemahnt: „Socialism [...] is not Russian; it is not Chine-

\footnotetext{
${ }^{97}$ Zu den Exilgruppen vgl. Cornelis van Dijk, The Indonesian Communist Party (PKI) and its Relations with the Soviet Union and the People's Republic of (hina, The Hague 1972.

98 Margot Light, Introduction, in: Dies. (Hrsg.), Troubled Friendships: Moscow's Third World Ventures, London 1993, S. 1-28, hier S. 13-15.

99 Aussage Georgij 1. Mirskijs laut Rubinstein, Red Star, S. 21 f.

100) David Ottaway/Mariana Ottaway, Afrocommunism, New York/London 1981; William H. Friedland/Carl G. Rosberg (Hrsg.), African Socialism, Stanford 1964. Zum sowjetischen Engagement in Afrika insgesamt vgl. Matusevich (Hrsg.), Africa in Russia; ders., No Easy Row for a Russian Hoe: Ideology and Pragmatism in Nigerian-Soviet Relations, 196()-1991, Trenton, N. J. 2003, leider ohne Nutzung der einschlägigen sowjetischen Archivdokumente.

101 Colin Legum, Pan-Africanism. A Short Political Guide, I ondon ${ }^{2} 1965$, S. 105.
} 
se. It is not African, or Asian or European - it is proletarian. " ${ }^{102}$ Von der vor allem in den 1960er Jahren dominierenden Auslegung, Sozialismus bedeute für Afrika, einfach zu den traditionellen Wurzeln der Gesellschaften (Ujamaa) zurückzukehren ${ }^{103}$, schwenkten etliche afrikanische Marxisten in den 1970er Jahren darauf um, doch die UdSSR als Vorbild eines für Afrika gangbaren Entwicklungsweges zu akzeptieren ${ }^{104}$. Zu diesem Zeitpunkt zählten die sowjetfreundlichen KPs Afrikas allerdings übersichtliche 60000 Mitglieder, was zwar einer Verzwölffachung seit 1939 entsprach, aber keineswegs zufriedenstellend war. ${ }^{105}$

Solche supranational ausgerichteten Modelle wie der Afromarxismus speisten sich aus einer postkolonialen Abwehrhaltung und waren oft noch mit weiteren universalistischen Wertvorstellungen gekoppelt wie dem Pan-Afrikanismus oder der pan-arabischen Bewegung. Diese waren wiederum oft mit religiösen Ideen durchsetzt ${ }^{106}$ wie bei einem der wichtigsten Theoretiker des Pan-Afrikanismus, George Padmore. ${ }^{107}$ Er betrachtete allerdings trotz seiner frühen Begeisterung und seines Aktivismus in der UdSSR unter anderem als Mitglied des Moskauer Sowjet den Pan-Afrikanismus als Lösung für Afrika, der sowohl den „westlichen“ Kommunismus als auch den afrikanischen Tribalismus entschärfe. ${ }^{108}$ Doch während im Afrika südlich der Sahara typischerweise eher nationalistische Motive in Konkurrenz zum Sozialismus standen, war in den arabischen Staaten insbesondere der Islam hemmend für die Verbreitung kommunistischer Ideen und Parteien. Der arabische Sozialismus hob nicht selten auf eine Vereinbarkeit von Religion und Politik ab, welche die atheistische Grundhaltung der KPdSU nicht bedienen konnte. Die meisten der arabischen KPs blieben daher verboten und wiesen oft nur wenige hundert oder tausend Mitglieder auf. ${ }^{109}$ Im Iran unterstützte die kommunistische Tudeh-Partei sogar den blutigen Wechsel vom Shah-Regime zur Theokratie Ajatollah Chomeinis. ${ }^{110}$

Im Bereich der Religion, speziell des Islam, zeigt das Beispiel Indonesien in den frühen 1960er Jahren mögliche Aushandlungsmechanismen zwischen sowjetischen Diplomaten und indonesischen Politikern. Dort bemühte sich Moskau auf Anraten Sukarnos und des PKI-Generalsekretärs, den Islam in die zwischenstaatliche Diplomatie einzubinden, um in Jakarta ein positives Bild der UdSSR zu verbreiten und der Bevölkerung eines der weltgrößten muslimischen Staaten die Furcht vor dem sowjetischen Atheismus zu nehmen. ${ }^{11}$

${ }^{102}$ Babu, African Socialism, S. 70f,; dagegen Léopold Sédar Senghor, African-Style Socialism, in: William H. Friedland/Carl G. Rosberg (Hrsg.), African Socialism, Stanford 1964, S. 264-266.

103 Vgl. beispielhaft die Ausführungen des Präsidenten von Tanganjika, Julius K. Nyerere, in: Ujamaa: The Basis of African Socialism, in: William H. Friedland/Carl G. Rosberg (Hrsg.), African Socialism, Stanford 1964, S. 238-247.

104 Ottaway/Ottaway, Afrocommunism, S. 13.

105 Für die Zahlenangaben vgl. Pyotr Manchkha, Africa on the New Road, Moskau 1972, S. 56.

106 Zum Pan-Afrikanismus und Padmores religiösen Ideen vgl. George Padmore, Pan-Africanism or Communism? The Coming Struggle for Africa, London 1956; Berner, Afrikapolitik, S. 727.

${ }_{107}$ Zu Padmore vgl. James R. Hooker, Black Revolutionary. George Padmore's Path From Communism to Pan-Africanism, New York 1967. Dazu kurz: Berner, Afrikapolitik, S. 727.

${ }_{108}$ Padmore, Pan-Africanism, S. 379. Zum Gesamtkomplex vgl. auch Constantin Katsakioris, L'Union Soviétique et les Intellectuels Africains. Internationalisme, panafricanisme et négritude pendant les années de la décolonisation, 1954-1964, in: Cahiers du monde russe 47 (2006), H. 1-2, S. 15-32, bes. S. 23-31; Matusevich, Ideology, S. 25 f.

109 Berner, Arabische Länder, S. 68 und 71. Dort auch Zahlen der KP-Mitglieder- und Sympathisantenstärken.

110 Tulsiram, The History of Communist Movement in Iran, Bhopal 1981, bes. S. 159-161.

111 Vgl. dazu Ragna Boden, Euro-asiatische Kulturdistanzen und ihre Überwindung. Ein Beitrag zur Kulturgeschichte der sowjetischen Diplomatie, in: Jahrbücher für Geschichte Osteuropas 56 (2008), H. 1, S. 54-71. 
Die Phase seit Mitte der 1960er Jahre war vor allem vom sowjetischen Engagement in Stellvertreterkriegen gekennzeichnet. Hier unterstützten der Westen und die sozialistischen Staaten jeweils ihre Favoriten mit Waffen und Know-how, so dass die politischen Frontstellungen nicht selten in Bürgerkriege ausarteten. ${ }^{12}$ In Indochina und besonders im Kambodscha-Konflikt kulminierten die Probleme sowohl des Ost-West-Konfliktes als auch des Bruchs innerhalb der kommunistischen Weltbewegung. Letztlich trugen die vietnamesischen Kommunisten, die Moskau näherstanden, den Sieg über die vom Westen und von Peking unterstützten Roten Khmer davon, wenn auch unter ungeheuren Opfern für sie selbst und die Bevölkerung in der Region insgesamt. Die Niederlage der USA gegen die vietnamesischen Kommunisten und das Scheitern des chinesischen Eingriffs zugunsten der ebenfalls vom Westen unterstützten „Steinzeit-Kommunisten“ der Roten Khmer bedeuteten einen Triumph sowohl für die vietnamesischen Kommunisten als auch für Moskau, das diese mit Material und Beratern unterstützt hatte. ${ }^{113}$

Dieser Erfolg blieb freilich regional begrenzt. Auch wurde wohl wahrgenommen, dass die Initiative von der KP des Entwicklungslandes ausgegangen war. Ein Kennzeichen dieser Zeit war, dass die außereuropäischen KPs zunehmend eigenständig handelten in ihrem Engagement für die globale Verbreitung des Kommunismus. Wo die sowjetische Führung gezögert hatte, die Revolution außerhalb Europas weiterzutreiben, ergriffen nun Fidel Castro und bis zu seinem Tod 1967 auch Che Guevara die Initiative in Afrika und Lateinamerika. Während die sowjetische Unterstützung in Angola sich nach der innerangolanischen und internationalen Lage richtete, hielten die Kubaner zur „Volksbewegung zur Befreiung Angolas“ (Movimento Popular de Libertação de Angola - MPLA) und betrieben ihre Unterstützungspolitik zunächst unabhängig von Moskau. ${ }^{11 \cdot 4}$ In Äthiopien dagegen arbeiteten Moskau und Havanna von Beginn an, spätestens seit 1977, eng zusammen. ${ }^{115}$ Ermutigt vom Erfolg in Angola und überzeugt vom geschickten Gebrauch der marxistischen Symbolik, unterstützte Moskau diese während der Brežnev-Phase wichtigste marxistisch inspirierte Revolution in Afrika. ${ }^{116}$

Konnte die globale Unterstützung der UdSSR den kommunistischen Parteien als Ausweis des aktiven Einsatzes der sozialistischen Supermacht für die Belange der Entwicklungsländer gelten, so hatte die zwischenzeitliche Annäherung der Supermächte seit Beginn der 1970er Jahre die KPs weltweit irritiert. Insbesondere die pro-chinesisch gesinnten lateinamerikanischen Genossen - bis auf Kuba - nahmen die sowjetischen Verständigungsversuche mit den USA zum Anlass, gegen den „russischen Sozialimperialismus" zu wettern und vor Moskaus Entwicklungshilfe zu warnen, die ihrer Ansicht nach als Instrument zur ökonomischen, politischen und strategischen Einflussnahme missbraucht werde. ${ }^{117}$

112 Im Zeitraum 1945-1985 fanden 95 Prozent (oder 145 von 152) aller kriegerischen Konflikte in der „Dritten Welt“ statt. Vgl. Gerald Braun, Kriege und Konflikte in der Dritten Welt, in: Dieter Oberndörfer/Theodor Hanf (Hrsg.), Entwicklungspolitik, Stuttgart u.a. 1986, S. 46-77, hier S. 47.

113 Strassner, Kambodscha-Konflikt, S.21; Ilya V. Gaiduk, The Soviet Union and the Vietnam War, Chicago 1996; Olsen, Soviet-Vietnamese Relations.

114 Peter Shearman, The Soviet Union and Cuba: The „Best“ of Friends, in: Margot Light (Hrsg.), Troubled Friendships: Moscow's Third World Ventures, London 1993, S. 166-190, hier S. 175.

115 Ebenda. Vgl. auch den Beitrag von Radoslav Yordanov in diesem Band, der den Beginn mit Mai 1977 anset.t.

$116 \mathrm{Vgl}$. auch Westad, Gilobal Cold War, S. 251-253.

117 Gemcinsame Erklärung marxistisch-leninistischer Parteien Lateinamerikas, Tirana 1977, S.7. 
Die erste offene sowjetische Militärintervention zugunsten der Kommunisten eines Entwicklungslandes 1979 bedeutete eine neue Qualität sowjetischer Politik. ${ }^{118}$ Das sowjetische Eingreifen war in diesem Fall nicht einmal vorwiegend ideologisch, sondern zu einem großen Teil geostrategisch begründet: Kabul lag im Vorfeld der sozialistischen Supermacht. Somit war es folgerichtig, die dortigen Kommunisten mit allen Mitteln zu unterstützen. Der Krieg in Afghanistan entwickelte sich jedoch - wie in Vietnam und Angola - zu einem Stellvertreterkrieg der Systeme mit entsprechend hohen Opferzahlen. Bezeichnenderweise scheiterte die UdSSR mit ihrer ersten derartigen Offensive aus eigener Initiative. Nicht nur die von den USA geförderten islamistischen Gegenkräfte boten Widerstand. Selbst die afghanischen Kommunisten schwächten ihre Stellung durch machtpolitische Auseinandersetzungen. Hieran hatte allerdings Moskau keinen geringen Anteil. ${ }^{119}$

\section{Die Auswirkungen der sowjetischen Politik des "neuen Denkens”}

Angesichts der immer dringlicher werdenden innenpolitischen Probleme versuchte Michail Gorbačev, die außenpolitischen Belastungen der UdSSR zu verringern und sich mit den Westmächten zu verständigen. Die Aussage sowjetischer Vertreter auf einer internationalen kommunistischen Versammlung 1988, die UdSSR habe die Beharrungskraft des Kapitalismus unterschätzt ${ }^{120}$, wurde von vielen als sowjetische Kapitulation vor dem feindlichen System in den internationalen Beziehungen. gedeutet. Hinzu kam, dass im Zuge einer sowjetischen Befreiung von den Unterstützungsverpflichtungen gegenüber marxistischen Bewegungen der Entwicklungsländer die materielle Hilfe für sie auf ein Mindestmaß reduziert oder sogar eingestellt wurde. Der neue Generalsekretär der KPdSU, der praktisch über keine Erfahrung in der Politik gegenüber der Dritten Welt verfügte ${ }^{121}$, beendete vor allem die offensichtlich gescheiterte sowjetische Intervention in Afghanis$\tan ^{122}$. Dabei kompromittierte er die dortigen Genossen - wie 1962 Chruščev die Kubaner -, indem er über ihren Kopf hinweg entschied, und auch noch öffentlich ihren Angaben zur eigenen militärischen und politischen Stärke widersprach. ${ }^{123}$ Gorbačev „afghanisierte“ den Konflikt durch den Abzug sowjetischer Truppen und gab so die dortige kommunistische Führung dem Zusammenbruch preis. Aus ähnlichen Motiven - der Verständigung mit dem Westen - übte Moskau Druck auf Vietnam aus, sich aus Kambodscha zurückzuziehen. ${ }^{124}$ Die UdSSR war damit und mit ihren inneren Reformen wie zu Chruščevs Zeiten zu

\footnotetext{
118 Scott McMichael, The Soviet-Afghan War, in: Robin Highham/Frederick W. Kagan (Hrsg.), The Military History of the Soviet Union, New York 2002, S. 259-274; Shirin Tahir-Kheli, The Soviet Union in Afghanistan: Benefits and Costs, in: Robert H. Donaldson (Hrsg.), The Soviet Union in the Third World: Successes and Failures, Boulder, Col./London 1981, S. 217-231; Heinrich Vogel (Hrsg.), Die sowjetische Intervention in Afghanistan: Entstehung und Hintergründe einer weltpolitischen Krise, Baden-Baden 1980. Vgl. auch den Beitrag von Bernhard Chiari in diesem Band.

119 Westad, Global Cold War, S. 299-326.

120) Richard F. Staar, Checklist of Communist Parties in 1988, in: PoC 38 (1989), H. 1, S. 47-68, hier S. 47 .

121 David E. Albright, The USSR a the Third World in the 1980s, in: PoC 38 (1989), H. 2-3, S. 50-70, hier S.67.

122. Vgl. Gorbačevs Rede vom 8.2.1988: Michail Gorbatschow, „Erklärung zu Afghanistan“, in: Ders., Reden und Aufsätze zu Glasnost und Perestroika, Bindlach 1989, S.610-614, hier S.611.

${ }_{123} \mathrm{Zu}$ den kompromittierenden Aussagen vgl. Staar, Checklist, S. 56.

12.4 Andrew/Mitrokhin, World, S. 476. Siehe auch Bernd Schäfers Beitrag hier im Band.
} 
einem riskanten und wenig verlässlichen Partner für die KPs der Entwicklungsländer geworden.

Diese Maßnahmen waren Ausdruck eines veränderten Verhältnisses der Reformkräfte innerhalb der KPdSU zur weltpolitischen Rolle der Partei. Unter Brežnev hatte die sowjetische Führung noch den Anspruch vertreten, dass Moskau das Zentrum der kommunistischen Weltbewegung bleibe. ${ }^{125}$ Diese Sichtweise widersprach derjenigen der Weltkonferenz der KPs von 1969, die kommunistische Bewegung habe kein Zentrum. ${ }^{126}$ Im Sinne des „neuen Denkens“ gab erst Gorbačev diesen Führungsanspruch der KPdSU faktisch auf und plädierte im Rahmen der kommunistischen Weltbewegung für eine „Einheit in der Vielfalt". ${ }^{127}$ Dies resultierte aus der Erkenntnis, dass die Welle der kommunistischen Machtübernahmen stagnierte. Ähnlich wie in der euphorischen Phase nach der Oktoberrevolution in Europa hatten die Marxisten unterschiedlicher politischer Richtungen im Dekolonisierungsprozess eine Chance zur schlagartigen Verbreitung des Marxismus(-Leninismus) gesehen. Doch eine automatische Hinwendung der neu entstehenden Staaten zum Kommunismus aufgrund eines antiimperialistischen Reflexes war nicht erfolgt. Dort, wo kommunistische Parteien die Macht übernahmen, hatten sie zum Teil jahrzehntelange Kämpfe hinter sich wie die KP Chinas. Oder sie übernahmen nur einen Teil des Landes, während der andere nicht-kommunistisch blieb wie in Korea und anfangs in Vietnam.

Zum Ende der UdSSR hin zählte die kommunistische Weltbewegung - ein Terminus, an dem die sowjetische Führung als politischer Leitlinie zumindest verbal bis zum Ende festhielt - knapp 100 kommunistische und Arbeiterparteien, inklusive „revolutionär-demokratischer Parteien“. ${ }^{128}$ Von diesen stammten 77 aus Entwicklungsländern (einschließlich Chinas), die meisten aus Amerika (27), gefolgt von Asien und der Pazifikregion (21), dem Nahen Osten und Nordafrika (16) sowie Afrika südlich der Sahara (13). Von diesen 77 Parteien waren 25 verboten, 16 zählten als regierend. ${ }^{129}$ Letztere verteilten sich auf die Regionen wie folgt: je sechs in Asien und Afrika (ohne Maghreb), je zwei in Amerika und dem Nahen Osten. Bedeutend bexüglich der Mitgliederzahlen war nach China, wo die KP laut offiziellen Angaben 47 Millionen zählte (entsprechend etwa 4,7 Prozent der Bevölkerung) ${ }^{130}$, die Vietnamesische Arbeiterpartei mit 2,1 Millionen Mitgliedern (3,6 Prozent), Kuba mit einer halben Million (5 Prozent) und Indien, wo die beiden KPs je etwa 450000 Mitglieder zählten (zusammen 0,1 Prozent).

Die Weltrevolution war damit nicht direkt den Dekolonisierungen gefolgt und lag in den meisten Entwicklungsländern in weiter Ferne. Erfolg wurde eher durch Kampf als

\footnotetext{
125 Timmermann, KPdSU, S.3f.

${ }^{126}$ Heinz. Timmermann, Moskau und der internationale Kommunismus: Von der Komintern zur kommunistischen Weltbewegung, Köln 1986 (BBIOSt, H. 10, 1986), S. 36.

127 Timmermann, KPdSU, S. 3-5.

${ }^{128}$ Staar, Checklist, S. 47. Folgende Zahlen, sofern nicht anders vermerkt, nach Staar, Checklist. Diese Zahlen sind Näherungswerte, zeigen aber die Tendenz auf, um die es hier geht.

${ }^{129}$ In Afrika südlich der Sahara zählten жu den regierenden KPs die in Angola, Benin, der Volksrepublik Kongo, Äthiopien, Mosambik und Simbabwe; in Amerika nur die kubanische und die nicaraguanische; in der Asien-Pazifik-Region die in China, Nordvietnam, Nordkorea, der Mongolei, Kambodscha und Laos; in der Region Naher Osten und Nordafrika die in Afghanistan und dem Jemen (Volksdemokratische Republik).

130) Zum Vergleich: In der UdSSR betrug das Verhältnis bei 19,5 Millionen Mitgliedern der KPdSU und einer Bevölkerung von 285 Millionen etwa 6,8 Prozent. Ähnliche Zahlen für die Jahre 1990 (Parteimitgliedschaft von knapp 18 Millionen) und 1989 (Bevölkerungszahl von mehr als 286 Millionen) nennt Hildermeier, Geschichte.
} 
durch Wahlen erreicht, und oft bedurfte es dazu ausländischer, nicht zuletzt sowjetischer Unterstützung. Ohnehin erwiesen sich außerhalb Europas nur wenige KPs als moskautreu; die meisten verfolgten eigene Interessen, etliche wandten sich Peking zu. Auch folgte die kommunistische Weltbewegung eigenen Gesetzmäßigkeiten, die Moskau kaum steuern konnte. Was in Osteuropa in den späten 1940er Jahren funktioniert hatte, ließ sich nicht im Weltmaßstab wiederholen.

Ohnehin war die kommunistische Weltbewegung in der Politik des „neuen Denkens“, die die Welt sicherer machen sollte, nachrangig, wenn nicht störend. In Gorbačevs Bemühen um einen Ausgleich mit ehemaligen Gegnern in kapitalistischen Ländern lässt sich auch die Verständigung mit Suharto einordnen. Ohne dass dieser Zugeständnisse hätte machen müssen, konnte er 1989 ein Freundschafts- und Kooperationsabkommen mit der UdSSR schließen. Dabei hatte er weder die Lage der indonesischen Kommunisten verbessert noch das Verbot der Verbreitung kommunistischer Ideen aufgehoben. ${ }^{131}$ Wieder einmal hatte die PKI gegenüber der pragmatisch orientierten sowjetischen Außenpolitik das Nachsehen. Damit steht die Geschichte ihrer Beziehungen zur UdSSR als Beispiel für rund 70 Jahre sowjetischen Umgangs mit Kommunisten in Kolonien und Entwicklungsländern. Moskau nahm dabei zwar eine globale Führungsrolle für sich in Anspruch, verstand es aber kaum, sie souverän auszufüllen. Die zahlreichen Wendungen der sowjetischen Innenund Außenpolitik sowie die Vielfalt der kommunistischen Weltbewegung erschwerten die Orientierung der kommunistischen Parteien am sowjetischen Vorbild. Charakteristisch war der stete Wandel in der Moskauer Politik gegenüber den ausländischen Genossen und ein fehlendes Gesamtkonzept.

131 Vgl. dazu den Artikel: RI Will Not Change Stance on Communism, in: Jakarta Post, 13.9. 1989, S. 1, und den Kommentar von Leonid Shegalow, UdSSR - Indonesien. 25 Jahre Pause, in: Neue Zeit 39 (1989), S.7f., sowie seine Versuche, die neue Annäherungspolitik zu rechtfertigen. 